\title{
Healthcare personnel's experiences using video consultation in primary healthcare in rural areas
}

\author{
Annette M. Johansson ${ }^{1}$, Inger Lindberg ${ }^{2}$ and Siv Söderberg ${ }^{3}$ \\ ${ }^{1}$ Department of Health Science, Division of Nursing, Luleå University of Technology, Luleå, Sweden \\ ${ }^{2}$ Adjunct Associate Professor, Department of Health Science, Norrbotten County Council and Division of Nursing, \\ Luleå University of Technology, Luleå, Sweden \\ ${ }^{3}$ Professor, Department of Nursing Sciences, Mid Sweden University, Campus Östersund, Sweden
}

\begin{abstract}
Background: Patients living in rural areas often need to travel long distances for access to specialist care. To increase access to specialist care, video consultation between patients in primary healthcare and specialist care has been used. In order for this new method to be developed and used to the fullest, it is important to understand healthcare personnel's experiences with this intervention. Objective: The aim of this study was to describe healthcare personnel's experiences using video consultation in their work in primary healthcare. Method: A mixed methods design was used, and the data were analysed using qualitative and quantitative analysis methods. Interviews were conducted with eight general practitioners and one district nurse, all of whom had conducted a video consultation with a patient and a specialist physician or a cardiac specialist nurse. After each video consultation, the participants completed a consultation report/questionnaire. Results: Healthcare personnel considered video consultation to provide quicker access to specialist care for the patient, and greater security when the video consultation encounter was conducted at their own primary healthcare centre. They considered video consultation an opportunity to provide education and for the patients to ask questions. Conclusion: Video consultation is a satisfactory tool for healthcare personnel, and the technology is a new, useful method, especially for the district nurses. Further, video consultation is an opportunity for healthcare personnel to learn. However, for it to work as an accepted method, the technology must function well and be user friendly. It must also be clear that it is beneficial for the patients and the healthcare personnel.
\end{abstract}

Key words: healthcare personnel; mixed methods; rural area; specialist care; video consultation

Received 24 August 2015; revised 10 August 2016; accepted 19 August 2016;

first published online 19 September 2016

Long-distance travel, together with a lack of nurses and physicians, can create an obstacle to care for residents in rural areas (Smith and Grey, 2009; Toledo et al., 2012). Distance is often a central reason for the inequity of access to specialist care in rural communities, and it makes care planning a

Correspondence to: Annette M. Johansson, Department of Health Science, Division of Nursing, Luleå University of Technology, SE-971 87 Luleå, Sweden. Email: annette.johansson @ltu.se

(C) Cambridge University Press 2016 challenge (Smith and Grey, 2009). Residents in rural areas often have no choice but to take an often uncomfortable, expensive, and long journey (Johansson et al., 2014b) that usually ends with a specialist visit of 5-10 min (Smith and Grey, 2009).

The use of video consultation (VC) can improve service for patients who live in rural areas (Harrison et al., 2006; Wesson and Kupperschmidt, 2013) and improve communication between primary and specialist care (Norman, 2006). VC may offer access to healthcare that might not be available to 
patients in rural areas, especially for elderly patients with restricted ability to travel (Richstone et al., 2006; Wesson and Kupperschmidt, 2013). It can also provide educational and training opportunities for the healthcare professionals involved in the VC (Bynum and Irwin, 2011).

To increase accessibility to specialist care for people living in rural areas, VC has been shown to be an acceptable alternative to a face-to-face meeting (Toledo et al., 2012). Information and communication technology has become less expensive, easier to use and more accessible, such that the potential for use of $\mathrm{VC}$ between patients and healthcare providers has increased (Qidway et al., 2008). Research (Johansson et al., 2014a; 2014c) has shown that residents in rural areas are amenable positive to VC. Access to specialist care, regardless of where the care is geographically situated, increases the quality of care. If a physical examination is necessary, the general practitioner (GP) can perform the examination, take a sample, or treat an ailment with the specialist physician (SP) providing guidance through VC (Johansson et al., 2014c). That means examinations at a distance allow for a more efficient use of the SP, which enables the time before diagnosis and treatment plan to be shortened, such that the care is more effective (Iakovidis et al., 2004). There is also an opportunity to have a follow-up meeting with the SP via VC (Johansson et al., 2014a).

Decisions to write referrals are often made when the GP considers that she/he no longer has the knowledge or the ability to help the patient (Sigel and Leiper, 2004). Being present during the VC encounter between the patient and SP can increase the GP's and nurses' knowledge and understanding of the patient's disorder (Johansson et al., 2014a). Implementation of $\mathrm{VC}$ also strengthens the collaboration between the SP and district nurse (DN) (Gordon, 2012). When SPs use medical language that could be difficult for the patient to understand, it is important that the $\mathrm{DN}$ is present during a VC to support and explain information to the patient (Johansson et al., 2014b).

The success of VC will depend on adequate compensation systems that would motivate physicians to provide VC services (Toledo et al., 2012). It is equally important to evaluate the costs and personnel resources before the implementation of $\mathrm{VC}$ as a new method for specialist consultation (Johansson et al., 2014b). Training and ensuring

Primary Health Care Research \& Development 2017; 18: 73-83 understanding of $\mathrm{VC}$ are considered essential (Hanssen et al., 2007). To increase the understanding of what is important when implementing new working methods such as VC; staff experiences using VC must be considered. Therefore, the aim of this study was to describe healthcare personnel's experiences of using $\mathrm{VC}$ in their work in primary healthcare (PHC).

\section{Methods}

\section{Context}

The study was conducted in the county of Norrbotten in the northern part of Sweden. The county of Norrbotten covers $25 \%$ of the area of Sweden and has a population of 250000 inhabitants, which is a distribution of 2.6 persons $/ \mathrm{km}^{2}$. The main hospital where most of the specialist care is provided is situated in the provincial town. This means many residents must travel long distances for specialist care. This research project was performed when the County Council of Norrbotten was planning to implement the use of VC encounters for specialist care in all 34 healthcare centres in the county. The distance from each PHC centre to the nearest hospital is between 23 and $246 \mathrm{~km}$.

\section{Design}

An explanatory mixed methods design was chosen to achieve the aim of the study. By using both qualitative and quantitative methodologies, a mixed methods design can incorporate the strengths from both methods (cf. Cresswell and Plano Clark, 2007). The design was used to obtain a more nuanced view of the healthcare personnel's experience of using VC in their work. The quantitative data were collected with consultation reports and the qualitative data by conducting individual interviews with the healthcare personnel.

\section{The intervention: $\mathrm{VC}$ at a distance in real time}

The project was conducted between January 2010 and February 2012 using VC encounters for patients in need of specialist care. Five PHC centres were included in the project, and patients with skin problems met a dermatologist at the main hospital via VC. The project also included VC between a cardiac specialist nurse and patients with heart disease. 
The patient and the GP could decide to have a VC encounter with the SP or dermatologist instead of sending a referral to specialist care. Likewise, the cardiac specialist nurse and patient could decide to have a follow-up meeting and a possible medication adjustment via $\mathrm{VC}$ encounter. The $\mathrm{VC}$ encounter was held within a few days to a week from the visit at the PHC centre. In the beginning of the project, VC was scheduled one morning each week and at a pre-determined time for each PHC. In VC between patients and the cardiac specialist nurse, the time for VC was decided by the cardiac specialist nurse, and a sampling was carried out before by the DN responsible for the patient at the $\mathrm{PHC}$ centre.

\section{Technology}

A video solution in the form of a PC-based system from Polycom ${ }^{\circledR}, \mathrm{CMA}^{\circledR}$ desktop video conferencing software was used in the project. For sending real-time video between the PHC centre and the specialist clinic, a standard Logitech ${ }^{\circledR}$ web camera (Logitech Europe SA, EPFL - Quartier de l'Innovation, Daniel Borel Innovation Center, Lausanne) was used. The SP (dermatologist) used a standard headset; the GPs, DN and patients used external speakers and a microphone for audio communication. A high-speed regional broadband network was used for the communication in the County Council's part of Lumiora.

\section{Participant and procedure}

Eight GPs and one DN, aged 31-70 years $(\mathrm{Md}=43)$, participated in the study. Inclusion criterion was having participated in VC encounters with a patient. The GPs and the DN had participated with a patient in a VC encounter; the SP and the cardiac specialist nurse were at the main hospital. Those GPs or DN who had filled in a consultant report received a letter of informed consent to participate in an individual interview about the VC encounter. The interviews and consulting report from SPs will be reported elsewhere.

\section{Data collection method}

\section{Consultation report}

A consultation report was developed with the following sections: technical functionality, reason for contact, supervision and outcomes of the consultation. There were also questions about the date of the consultation which involved the PHC centre and the consulting physician's satisfaction with the VC encounter. An opportunity for a free text answer was also provided in the consultation report. After the first consultations, the GPs gave feedback on the consultation report in order to improve the understanding of the issues and answers. After the feedback, one alternative to a question was added to make the answer to the question more detailed. As 130 consultations reports were completed, and there were 174 consultations, the response rate was $75 \%$.

\section{Interviews}

Individual interviews were performed with the GPs and the DN using an interview guide. The interviews were guided by semi-structured questions; 'Could you please tell me your experiences using VC in your work'; 'Please tell me how it affected your work'; 'What are your thoughts about the new working method?'; 'Have you received any guidance or training via VC? If yes, can you please tell me about your experiences'; 'Please tell me about possibilities and obstacles you met when VC'; 'Do you experience that VC is something that gives increased accessibility to care for your patients?' Clarifying questions were used, that is, 'Can you give an example?'; 'What did you feel?' The interviews were carried out between December 2011 and June 2012, lasted between 10 and $30 \mathrm{~min}$ $(\mathrm{Md}=20)$ and were performed at the PHC centre or via phone. The interviews were audio taped and later transcribed verbatim.

\section{Data analysis}

\section{Quantitative analysis}

The consultation reports were analysed with descriptive statistics was used to calculate ordinal data (cf. Djurfeldt et al., 2008). The Statistical Package for Social Sciences (IBM SPSS ${ }^{\circledR}$ ), version 22.0 was used for the analysis.

\section{Qualitative content analysis}

The interviews were analysed by using qualitative content analysis (cf. Downe-Wamboldt, 1992). The methodology provides a systematic and objective way to come to meaningful conclusions 
and to describe a specific phenomenon of verbal communication, visual or written data. The analysis started by reading the transcribed text several times in order to achieve a sense of the content. Guided by the aim of the study, units of analysis including words, sentences, phrases, paragraphs, or whole text were identified and given a code, for example, patient, evaluation or technology. The encoded units of analysis were condensed and sorted into categories based on similarities and differences in the content, and those categories were then subsumed into seven categories. Finally, the authors reread the units of analysis and checked for the accuracy of their categorisation.

\section{Results}

The results are presented in two parts. Part I presents the results from the quantitative analysis of the consultation reports, and part II presents the results from the qualitative analysis of the interviews. In the presentation, healthcare personnel represented were GPs and DNs at the PHC centre, and SP represents the SP (ie, dermatologist).

\section{Part I: the result of the analysis of the consultation reports}

The most common cause for the GPs to be in need of SP consultation for the patient's health problem was diagnostic uncertainty $(75.2 \%)$. The second reason was to obtain advice on how to handle the health problem $(45.3 \%)$ and, in some cases, the GP needed both a diagnosis and advice regarding how to manage it. During the VC, GPs could obtain guidance from the SP on how and where to take a sample (eg, sample excision or a biopsy) (Table 1).

The reason for $\mathrm{VC}$ between the $\mathrm{DN}$ and specialist nurse was medical follow-up.

The technology used in VC mostly functioned well or very well (Tables 2 and 3 ).

Table 1 Reason for video consultation $(n=130)$

\begin{tabular}{ll}
\hline Reason for consultation & Frequency $[\%(n)]$ \\
\hline Diagnostic uncertainty & $75.2(88)$ \\
Advice on handling the problem & $45.3(53)$ \\
Second opinion & $24.8(29)$ \\
Guidance for sampling & $15.4(18)$ \\
\hline
\end{tabular}

Valid percentage.

Primary Health Care Research \& Development 2017; 18: 73-83
Table 2 Technical functionality [general practitioner $(n=130)]$

\begin{tabular}{lcclll}
\hline & $\begin{array}{l}\text { Very bad } \\
{[\%(n)]}\end{array}$ & $\begin{array}{l}\text { Bad } \\
{[\%(n)]}\end{array}$ & $\begin{array}{l}\text { Well } \\
{[\%(n)]}\end{array}$ & $\begin{array}{l}\text { Very well } \\
{[\%(n)]}\end{array}$ & $\begin{array}{l}\text { Total } \\
(n)\end{array}$ \\
\hline Audio & $5.8(6)$ & $3.3(3)$ & $54.5(62)$ & $36.4(41)$ & 112 \\
Picture & 0 & $14.2(17)$ & $65.8(75)$ & $20.0(20)$ & 112 \\
Colour & 0 & $10.0(12)$ & $71.7(80)$ & $18.3(19)$ & 111 \\
Sharpness & 0 & $16.8(20)$ & $65.6(73)$ & $17.6(17)$ & 110 \\
\hline
\end{tabular}

Valid percentage.

Table 3 Technical functionality [district nurse $(n=9)$ ]

\begin{tabular}{llllll}
\hline & $\begin{array}{l}\text { Very bad } \\
(n)\end{array}$ & $\begin{array}{l}\text { Bad } \\
(n)\end{array}$ & $\begin{array}{l}\text { Well } \\
(n)\end{array}$ & $\begin{array}{l}\text { Very well } \\
(n)\end{array}$ & $\begin{array}{l}\text { Total } \\
(n)\end{array}$ \\
\hline Audio & 1 & 1 & 4 & 3 & 9 \\
Picture & 1 & & 4 & 4 & 9 \\
Colour & & & 6 & 3 & 9 \\
Sharpness & & 5 & 4 & 9 \\
\hline
\end{tabular}

The results show that $15.4 \%$ of the patients received a referral to a specialist after VC. The reason for this may be that the patient needed treatment with potent medications that required sampling, information, and instructions. Another reason was that the SP could not attain enough information from the video picture to formulate a diagnosis. A majority of the patients $(62.3 \%)$ who had met the SP via VC could have a follow-up meeting at their PHC centre, and $11.9 \%$ considered that they were not in need of further treatment or follow-up. There were also patients who would meet the SP one more time $(6.0 \%)$ via VC for a follow-up of the treatment. Most of the GPs and DN who had used VC in their work were very pleased $(49.2 \%)$ or pleased $(39.2 \%)$ with the consultation; and $9.1 \%$ of the GPs were rather pleased. Only three GPs $(2.5 \%)$ were dissatisfied with the method. The cause of that were technical problems and having to take care of a patient that was late to the $\mathrm{VC}$ and therefore it became very stressful. The VC lasted between 5 and $30 \mathrm{~min}(\mathrm{Md}=15 \mathrm{~min})$.

\section{Part II: the results of the analysis of the interviews}

The analysis of the interviews revealed the following categories: advantage and disadvantages for the patient, advantages and disadvantages for 
healthcare personnel, the personal encounter, future possibilities for VC encounter, satisfaction with $\mathrm{VC}$ as a consultation method and opinions about the technology. The categories are presented below and illustrated by quotes from the participants.

\section{Advantage and disadvantages for the patient}

The GPs and DN experienced that patients were grateful for the opportunity for a VC encounter. VC together with their GP was considered a relief, more secure and comfortable for the patient. Healthcare personnel believed that the more rapid contact with the SP through VC was perceived by the patient as though the treatment was progressing. The GP expressed that even if it sometimes was a preliminary assessment of the patient's problem, the patient did not have to wait for months for a consultation at the main hospital.

The convenience, that the patient comes here and we are talking at once with the specialist at the other side of the computer, the ease of reaching the specialist. Availability has improved as the patient doesn't have to wait for three months. At least we have a preliminary assessment; it's the most important.

(GP7)

The GPs and DN experienced the availability of a VC encounter with the SP as sufficient, as it was possible to have a meeting every week and two meetings on the same day if needed. Samples could be taken in advance at the PHC centre, and the SP could have the test result at the VC encounter and the encounter could be completed in a shorter time.

Healthcare personnel described that patients who participated in VC encounters were positive and considered it amazing that they did not have to travel far for a meeting with a SP or a nurse. General practitioners described that before the opportunity there were occasions when they tried to treat the patient's problem without consultation to avoid any unnecessary travel for the patient.

The patient can avoid the long journey. Not all patients are capable of those travels, and it makes it easier for me to decide on a skin consultation [VC]. Sometimes I've decided not to involve the dermatologist at all and tried entirely by myself instead.

Healthcare personnel expressed that it was not only the trip that could be troublesome for the patient. They also considered it expensive for the patients, as relatives might have to follow as company, and waiting for transport could be burdensome. According to the GPs, the patient was pleased to obtain a second opinion and confirmation from the SP via the VC encounter. The GP and DN had to talk with both the patient and the SP/cardiac specialist nurse.

\section{Advantages and disadvantages for the healthcare personnel}

The GP described that the long distances to the SP often meant that they treated the patients even though they were not sure of the diagnosis. VC was considered by the GPs as a valuable opportunity to conduct a clinical discussion with the SP, and thus be able to make a diagnosis. General practitioners experienced $\mathrm{VC}$ as a learning opportunity, and they considered their knowledge as increasing. A second opinion was considered valuable when the GP's own knowledge regarding diagnosis and treatment did not help the patient. Response to a referral may have resulted in new questions, and that meant extra work and not the exchange of knowledge as happened via VC. Additionally, telephone contact with the SP was considered unsatisfactory when skin alterations may be difficult to describe clearly in words. The GPs described that they wrote fewer referrals when they had the opportunity for a VC encounter. They expressed that nearly every VC meant one referral fewer.

From my part, the referrals have decreased. I do not remember the last time I sent a referral.

(GP1)

Healthcare personnel expressed that VC saved time for them. There were also healthcare personnel that expressed that it took time because they needed to see the patient again for the VC, which was performed only on a certain day and time. The DN expressed that when they participated in $\mathrm{VC}$, and the encounter was scheduled with the cardiac specialist nurse, the patient came to the Primary Health Care Research \& Development 2017; 18: 73-83 
PHC centre one time just for the VC. General practitioners considered it quicker to write a referral than to arrange a VC. They also felt that the diagnosis and treatment for the patient's problem could be delayed if it was not possible to obtain a diagnosis during $\mathrm{VC}$, and the patient had to travel for a face-to-face encounter with the SP. General practitioners and DN expressed that to maintain knowledge and interest for $\mathrm{VC}$, more specialist areas and more frequent VCs were needed. One GP considered that because the patient attended a VC, it was the SP's recommendations that were heeded, even if the GP did not agree, because the SP had competency in the specialty. When different opinions between the GP and SP arose, the GP thought it could be because the SP could not touch the patient's skin lesion.

If you think entirely different [from the SP], or if the dermatologist thinks and considers to do so anyway and it's a consultation with the patient, then you have to be humble and buy everything that is said. It feels like it leaves no room for me to make any of my own modifications of what we hear, but it will be what the dermatologist says, whether I agree or not.

(GP5)

\section{The personal encounter}

Healthcare personnel described that the VC encounter meant multi-person communication in which everyone involved had the opportunity to be heard. At times, when the GP had to interpret because the patient could not speak Swedish, the VC encounter was experienced as complicated, but not impossible. Healthcare personnel described that if the patient had headphones because of impaired hearing; the encounter was experienced as more inconvenient. Some GPs expressed that although the VC encounter not was the best solution, it was an adequate alternative to a phone call. Although VC encounters were a good solution for accessibility, some considered meeting the SP via a computer less adequate than face-to-face contact. One GP expressed that what was the best alternative was if the SP came to the PHC centre.

\section{Future possibilities for VC encounter}

Healthcare personnel expressed that the use of VC would increase as they became aware of the

Primary Health Care Research \& Development 2017; 18: 73-83 possibility, and that it was implemented in an increasing number of clinics and PHC centres. They considered that the more experience healthcare personnel had with the technology and the greater importance of accessibility, the use of $\mathrm{VC}$ would come to mean fewer face-to-face visits to the SP. The opinion of the GP was that $\mathrm{VC}$ was a costeffective solution for rural areas, mainly because all of the other options would be too expensive.

I think it will be a very cost effective solution for rural areas [...] where there is real rural area, it's the future I think, and everything else is too expensive.

(GP1)

General practitioners considered that there could be money saved if GPs could participate in $\mathrm{VC}$ in order to learn when another GP and patient had a VC encounter with the SP. They stated that there were several areas, besides dermatology, which were suitable for VC: in orthopaedics follow-ups through VC with X-ray images of fractures; within surgery (eg, minor local surgeries that can be performed at the PHC centre); within ophthalmology, equipment for eyes and ear diagnostics are available at PHC centres, but for VC, they required a camera connected to the eye microscope; within psychiatry, for patients who do not want to go to a psychiatry clinic but are at risk of suicide, instead of compulsory care they have the opportunity to talk to a psychiatrist via VC encounter. DNs considered that there should be the possibility for the DN to perform a VC from the patient's home with the GP at the PHC centre.

The right thing is that you bring it [VC equipment] and can make contact with a doctor immediately from the patients' home without having to go to a visit. You can meet via the computer, and you can send the ECG to the healthcare centre.

There were even ideas for special days when the PHC should gather patients in a particular field (eg, dermatology) and connect to the dermatologist via VC. Consultation between on-call nurses in rural areas and physicians in emergency care were considered important as it sometimes was impossible to obtain access to an on-call physician in rural areas. 
Satisfaction with VC as a working method

Healthcare personnel felt that VC was a good working tool and believed that there were many opportunities to develop the use of technology. They emphasised that it was important that the technology worked well in order to have a comfortable VC encounter. Everyone involved could participate in a discussion about the patient's problems and treatment, and no referrals or responses needed to be sent. Healthcare personnel were in agreement that it was a great advantage that the patient, GP, DN and SP could talk to each other simultaneously.

Thus, the advantage you have is that you are talking all together in the same room. The patient is also there and can follow the reasoning if we discuss the ailment or disease or whatever it is so it can be an advantage it is quite clear.

(GP4)

It was considered important to have the opportunity to practice with the technology and to obtain positive feedback in order for the working method not to be perceived as a burden. There were also GPs who could not see any advantages of using VC besides early in their career for educational purposes. General practitioners felt that there were limitations and that the SPs could not use their full abilities via VC, but it was still considered important to use VC and try to develop the technology.

\section{Opinions about the technology}

General practitioners considered it difficult to describe something (eg, redness and scaly skin) via referral and felt that VC was the next step in consulting a SP, but doubts were also expressed over the quality of the picture the SP received through VC. They requested a better camera for a clearer picture, and the light in the examination room was also considered to cause blurred images on some occasions. Those who had difficulties with poor pictures or sound thought these problems were something that could be solved without major problems. Some of the GPs thought that the technique was simple, although they had not tested it earlier and/or been prepared to have a VC. General practitioners considered that the technology should be simplified; there were too many steps before the VC would be regarded as user friendly and safe.

What so ever, we need the technology to be simplified. There are too many choices and too much that can go wrong today.

General practitioners considered that there was no coordinating structure among $\mathrm{VC}$, video conference, and dictation equipment, and there were too many various actions that would have to be carried out depending on what part of the technology would be used. They considered that equipment mostly had good user manuals, but when the equipment used advanced technology and tight scheduling, there was no time to read and understand the instructions. The GPs expressed that they had lost dictated data due to the change between different programs in the computer, from VC to Dictaphone, that had not been carried out or not been performed properly.

\section{Thoughts about organisation and VC}

Healthcare personnel considered that the VC did not require many organisational changes. According to the GPs, there was no significant difference between VC and writing a referral, they told the SP what the problem was, the SP told them how to treat the patient's problem or asked the patient to come to the clinic. For the GP, it meant shortened processing time and was administratively simple.

There is no big difference, (to me organisationally). Usually I have written a referral to the dermatology clinic and either they answered and described how to treat the patient or they wanted to see the patient at the clinic.

(GP5).

The GP, DN and SP have access to the Electronic Health Records that is shared in the region. It was considered as time saved when the SP had the opportunity to read about the patient's problem, medical treatment and test results before the VC. A prerequisite was that the notes in the patient's medical record were sufficiently informative. There were also opinions that VC took time, but the GPs thought that it was

Primary Health Care Research \& Development 2017; 18: 73-83 
a service they wanted to give their patients. They considered that the organisation had to change so that the patient did not have to come back for another appointment to participate in VC. General practitioners thought that they either booked a new appointment for $\mathrm{VC}$, or the specialist care needed to change their organisation so there would be better accessibility to specialist care. It was considered by the GPs as important that the VC not mean extra work for them. They had an idea that the SP on call could participate in $\mathrm{VC}$, in that case, the patient did not have to come back for another appointment.

There were some considerations among the GPs about the pre-determined time for VC that was on forenoon. The majority of them had their telephone time at that time, so they had to cancel it or try to plan for a time that suited both the patient, SP and GP. They considered that greater access to the SP would imply that VC became more resource saving. General practitioners expressed that another hindrance was if the patient was in need of an interpreter. It was difficult to find an interpreter within so short time, and it was considered to take more time as there was need for more questioning and answers, which is in contrast to consultations without an interpreter.

It's difficult if the patient comes on Monday and the consultation is on Tuesday. It may be very difficult to find an interpreter at eight o'clock in the morning. If they [the patients] speak English that is fine, then you can considerer you have 20 minutes consultation [VC] but with the interpreter you have ten minutes since you have to ask and answer, it takes time.

(GP3)

General practitioners considered that the calendar for bookings of VC times was a good tool, as they could make an appointment for the VC when the patient sat beside them. One GP thought that the calendar was troublesome because they had to change the setting of the clinics name and remember yet another code. General practitioners considered it convenient that the DNs booked appointments' for VC. They were considered to be the one who had the best overview of the reservations, especially when there was relay physicians who might not be familiar with VC.

Primary Health Care Research \& Development 2017; 18: 73-83
It is our coordinating DN who has booked this appointment, so she is like the spider in the net and she has control of appointments.

(GP8)

General practitioners considered it troublesome if it was only one DN who took care of the appointments for VC and she was not available when the GP wanted to give the patient an appointment when he or she was at the PHC centre.

General practitioners considered it important that there was a room that was available for the $\mathrm{VC}$ if it could not take place at the GPs office. There were PHC centres that had VC at the nurses' expedition, and this was not considered optimal for the patient, especially if the patient's problem necessitated them to undress. The GPs and DN thought that it was the organisation at the PHC that should arrange for a room with good equipment for VC. The GPs that only attended the VC and did not know how the system was used meant that it was important that more than one DN was able to manage the system. That was the opinion, not only from the relay physicians, but also from regular GPs in this study. The DNs also had the responsibility to run tests on the patient in time so the results were available before the VC.

\section{Discussion}

This study shows that healthcare personnel experienced that the patients considered VC with their GP or DN secure and comfortable, even if it was a preliminary assessment of their health problem. VC can improve access to healthcare services and skilled healthcare personnel and reduce stress, travelling time, and expenses for the patients (Johansson et al., 2014a). In our study, GPs described that before the use of VC, they tried to treat the patient to avoid unnecessary travel for the patient to the SP. Even when there was no doubt about diagnosis and treatment, the patient sometimes wished to obtain a second opinion from the SP via VC encounter. Ireson et al. (2009) described that the experience of having a referral to a SP was obviously important for the patients. When they did not receive the care they considered themselves needed, they thought that one of the reasons was that their GP was unwilling to write a referral to a SP (Johansson et al., 2014c). 
A referral to an SP requires coordination of care. Thus, PHC and specialist care are usually performed in different settings, and communication and shared information between them are identified as a prerequisite for providing faultless integrated care (Tarrant et al., 2008). However, it appears that patients' expectations of a faultless transition often are not met by healthcare personnel (Sanchez-Piadra et al., 2014). The results from this study show that some of these problems may be overcome if the patient has the possibility of a second opinion from the SP via VC.

General practitioners experienced $\mathrm{VC}$ as a learning opportunity, and the GPs who had access to VC encounters wrote fewer referrals to the SP. For example, the GP could obtain guidance from the SP on how to take a sample (eg, sample excision or a biopsy). Moffat and Eley (2010) described that the direct benefits for rural healthcare personnel is the ability to provide an enhanced local service, and that the indirect benefits occur through experiential learning from close contact with the SP in clinic work. Furthermore, as a consequence, the healthcare personnel perceived reduced isolation, improved communication, and increased skill and confidence. Our study revealed that VC also meant multi-person communication in which everyone involved had the opportunity to be heard. This does not echo the results in Miller (2003), where the patients were the least active participants, making the fewest comments, asking the fewest questions, and exerting the least control. Not only may patients participate to a lesser extent, but there are also concerns that healthcare personnel and SPs may communicate with each other to the exclusion of the patient. If that happens, it may decrease patients' trust and satisfaction. Also Agha et al. (2009) considered that the physicians control the conversation and that the patient assumes a more passive role. In order to carry out a VC with the patient, instead of about the patient, Johansson et al. (2014c) noted the importance of $\mathrm{VC}$ to be patient centred. Therefore, it is particularly important that the SP speak directly to the patient and not solely to the GP or the DN.

The results show that the healthcare personnel experienced that VC was a good working tool and believed that there were many opportunities to develop the use of technology. However, de Veer et al. (2011) described that when the nurses thought that the patient would benefit from the new technology, they were more willing to actually use it. In contrast, when the expected benefits from the patient were thought to be low or unclear, this impeded the introduction of the technology. Korzep (2010) described that the SP had the possibility to see more patients over a wider area, and that this could affect the need for physicians because the patient-to-physician relationship could increase. There would still be a need for SPs and GPs, but there could be fewer of them as they would be able to reach out to a wider area. The shortage of physicians that currently exists may be solved when they have the opportunity to cover for each other.

Social interaction is important in VC but when there is a need for diagnostic of a skin problem, also the picture quality is very important. The results in our study revealed that difficulties, for example, poor picture or sound, were considered as something that could be solved without major problems. Nevertheless, the GPs also considered that the technology should be simplified. The technology meant too many various procedures were to be carried out depending on what part (eg, Dictaphone, VC) of the technology was be used. Norum, et al. (2007) noted that the technology should be user friendly, and that there should be a plan for upgrading the technology and keeping it up to date. Miller (2011) argued that patients may appreciate VC more than healthcare personnel because they can see the benefit immediately when they do not have to travel or change their schedule. Healthcare personnel, on the other hand, may need to see greater benefit, or it may be viewed as an unnecessary intrusion in their practices.

Healthcare personnel thought that the implementation/use of VC would not require many organisational changes, although, a literature review, Stenlund and Mines (2012) showed that organisations ought to consider their level of preparedness when planning for VC. Every stakeholder should be involved in developing the plan for implementation. Organisations must make sure that the workplace is prepared for the new working method by examining human resources readiness, technological readiness and training and education of the staff. Matusitz and Breen (2007) considered that there had often been medical doctors that have dominated the field of telemedicine, but the role of nurses in the field has

Primary Health Care Research \& Development 2017; 18: 73-83 
now been expanded, and it pervades the telemedicine sphere. Harrison and Lee (2006) described that the nursing role within the ever-growing e-health market assumes that nurses have knowledge of everything from how the system works to being an expert on how the technology is used, and it requires additional basic education and continuing training.

\section{Methodological considerations}

The number of participants in this study might be considered low, but the results are based on numerous consultations $(n=130)$, distributed among PHC centres located throughout the county. Therefore, the results can be considered representative of the PHC centres in rural areas. Data were similar, so it is probable that the result would not change with a larger group of participants. The shortest interview lasted $\sim 10$ min, but the content was substantial.

The validity of the consultation reports as confirmed by a pilot test, and reliability was achieved as the questions consisted of pre-determined questions so there would not be opportunities for misinterpretation. The results of the qualitative analysis from the interviews are possible to transfer to similar circumstances, such as VC within other healthcare areas, for example, between patients in home care and DNs. According to Sandelowski (2000), linking the result of each data technique could be achieved by using the techniques usually used with the data. After analysing the data separately, the data are combined at the interpretive level, but each data set remains analytically separate from the other.

The strength with mixed methods design is that it is particularly well suited to develop different forms of interventions. According to Cresswell and Plano Clark (2007), this method is suitable to use as it provides the opportunity to strengthen the results. The results from qualitative data analysis can also help to explain the results of the quantitative analysis.

\section{Conclusion}

VC is a very useful working tool for healthcare personnel to support patients and to increase Primary Health Care Research \& Development 2017; 18: 73-83 accessibility to specialist care. It can also be used between DNs and patients in rural areas. The technology creates a new working method, especially for the DNs. VC is an opportunity for healthcare personnel to learn from the SP and GP, and that ultimately leads to better trained healthcare personnel. However, for VC to work as an accepted working method, the technology must function well and be user friendly. It must also be clear that VC benefits the patients and the healthcare personnel.

\section{Acknowledgements}

This study was supported by the Department of Health Science and was funded by 2 nd goal EU contributions through the e-Health Innovation Centre (EIC) at Luleå University of Technology, Luleå, Sweden. The authors thank the healthcare personnel for participating in this study.

\section{Ethical Standards}

Oral and written informed consent to participate in the study was obtained from the participants. They were guaranteed confidentiality and an anonymous presentation of the results. The study was approved by the Regional Ethical Board (dnr. 2010-5-31).

\section{Conflicts of Interest}

The authors declare that there is no conflict of interests regarding the publication of this paper.

\section{References}

Agha, Z., Schapira, R.M., Laud, P.W., McNutt, G. and Roter, D.L. 2009: Patient satisfaction with physician-patient communication during telemedicine. Telemedicine Journal and e-Health 15, 830-39.

Bynum, A.B. and Irwin, C.A. 2011: Evaluation of the consultant characteristics on telemedicine diagnosis and treatment. International Journal of Telemedicine and Applications 11, $1-8$.

Cresswell, J.W. and Plano Clark, V.L. 2007. Choosing mixed methods research. Thousand Oaks, CA and London and New Delhi: SAGE Publications.

de Veer, A.J.E., Fleuren, M.A.H., Bekkerna, N. and Francke, A.L. 2011: Successful implementation of new technology in nursing care: a questionnaire survey of nurse users. BMC Medical Informatics and Decision Making 11, 67. 
Djurfeldt, G., Larsson, R. and Stjärnhagen, O. 2008. Statistisk verktygslåda - samhällsvetenskaplig orsaksanalys med kvantitativa metoder (Statistical toolbox - social scientific causal analysis with quantitative methods). Lund: Studentlitteratur AB.

Downe-Wamboldt, B. 1992: Content analysis: method, applications and issues. Health Care for Women International 13, 313-21.

Gordon, J. 2012: Dermatologic assessment from a distance: the use of teledermatology in an outpatient chemotherapy infusion center. Clinical Journal of Oncology Nursing 16, 418-20.

Hanssen, B., Wangberg, S.C. and Gammon, D. 2007: Use of videoconferencing in Norwegian psychiatry. Journal of Telemedicine and Telecare 13, 130-35.

Harrison, J.P. and Lee, A. 2006: The role of e-health in the changing health care environment. Nursing Economics 24, 283-89.

Harrison, R., MacFarlane, A., Murray, E. and Wallace, P. 2006: Patients' perceptions of joint teleconsultations: a qualitative evaluation. Health Expectations 9, 81-90.

Iakovidis, I., Wilson, P. and Healy, J.C. 2004. E-health: current situation and examples of implemented and beneficial e-health applications. Amsterdam and Oxford: IOS Press.

Ireson, C.L., Slavova, S., Steltenkamp, C.L. and Scutchfield, F. D. 2009: Bridging the care continuum: patient information needs for specialist referrals. BMC Health Service Research 9, 1-12.

Johansson, A.M., Lindberg, I. and Söderberg, S. 2014a: Patients' experiences with specialist care via video consultation in primary healthcare in rural areas. International Journal of Telemedicine and Applications 14, 1-7.

Johansson, A.M., Lindberg, I. and Söderberg, S. 2014b: The views of health-care personnel about video consultation prior to implementation in primary health care in rural areas. Primary Health Care Research \& Development 15, 170-79.

Johansson, A.M., Söderberg, S. and Lindberg, I. 2014c: Views of residents of rural areas on accessibility to specialist care through videoconference. Technology and Health Care 22, 147-55.

Korzep, K. 2010: The future of technology and the effect it may have on replacing human jobs. Technology and Health Care 18, 353-58.

Matusitz, J. and Breen, G.-M. 2007: Telemedicine: its effects on health communication. Health Communication 21, 73-83.

Moffat, J.J. and Eley, D.E. 2010: The reported benefits of telehealth for rural Australians. Australian Health Review 34, 276-81.
Miller, E.A. 2003: The technical and interpersonal aspects of telemedicine: effect on doctor-patient communication. Journal of Telemedicine and Telecare 9, 1-7.

Miller, E.A. 2011: The continuing need to investigate the nature and content of teleconsultation communication using interaction analysis techniques. Journal of Telemedicine and Telecare 17, 55-64.

Norman, S. 2006: The use of telemedicine in psychiatry. Journal of Psychiatric and Mental Health Nursing 13, 771-77.

Norum, J., Pedersen, S., Størmer, J., Rumpsfeld, M., Stormo, A., Jamissen, N., Sunde, H., Ingebrigtsen, T. and Larsen, M.-L. 2007: Prioritisation of telemedicine services for large scale implementation in Norway. Journal of Telemedicine and Telecare 13, 185-92.

Qidway, W., Beasley, J.W. and Gomez-Clavelina, G. 2008: The present status and future role of family doctors: a perspective from the international federation of primary care research network. Primary Health Care Research \& Development 9, 172-82.

Richstone, L., Kavoussi, L.R. and Lee, B.R. 2006: Telemedicine proven results and promise for the future. Contemporary Urology 10, 20-26.

Sanchez-Piedra, C.A., Prado-Galbarro, F.J., Garcia-Pérez, S. and Santamera, A.S. 2014: Factors associated with patient satisfaction with primary care in Europe: results from the EU prime care project. Quality in Primary Care 22, 147-55.

Sandelowski, M. 2000: Focus on research methods. Combining qualitative and quantitative sampling, data collection and analysis techniques. Research in Nursing \& Health 23, 246-55.

Sigel, P. and Leiper, R. 2004: GP views of their management and referral of psychological problems: a qualitative study. Psychology and Psychotherapy 77, 279-95.

Smith, A.C. and Gray, L.C. 2009: Telemedicine across the ages. The Medical Journal of Australia 190, 15-19.

Stenlund, D. and Mines, B. 2012: Videoconferencing and dietitian services in rural Ontario communities. Revue Canadienne de la Pratique et de la Recherche en diététique 73, 176-80.

Tarrant, C., Colman, A.M. and Stokes, T. 2008: Past experience, 'shadow of future', and patient trust: a cross sectional survey. British Journal of General Practice 58, 780-83.

Toledo, F.G., Triola, A., Ruppert, K. and Siminerio, L.M. 2012: Telemedicine consultations: an alternative model to increase access to diabetes specialist care in underserved rural communities. JMIR Research Protocols 1, e14.

Wesson, J.B. and Kupperschmidt, B. 2013: Rural trauma telemedicine. Journal of Trauma Nursing 20, 199-202. 